Cahiers de géographie du Québec

\title{
Commentaires, par Antoine Bailly, Paul Claval, Daniel Dory, Jean-Paul Ferrier, Yves Guermond, Christian Kesteloot, Eleonore Kofman, Jean-Bernard Racine, Claude Raffestin, Marie-Claire Robic.
}

Volume 32, numéro 87, 1988

URI : https://id.erudit.org/iderudit/021985ar

DOI : https://doi.org/10.7202/021985ar

Aller au sommaire du numéro

Éditeur(s)

Département de géographie de l'Université Laval

ISSN

0007-9766 (imprimé)

1708-8968 (numérique)

Découvrir la revue

Citer cet article

(1988). Commentaires, par Antoine Bailly, Paul Claval, Daniel Dory, Jean-Paul Ferrier, Yves Guermond, Christian Kesteloot, Eleonore Kofman, Jean-Bernard Racine, Claude Raffestin, Marie-Claire Robic. Cahiers de géographie du Québec,

32(87), 327-347. https://doi.org/10.7202/021985ar d'utilisation que vous pouvez consulter en ligne. 


\section{RÉPLIQUES EUROPÉENNES}

\section{Antoine BAILLY (Université de Genève)}

La géographie a changé; tous les articles l'illustrent. Mais que de cheminements semblables de part et d'autres de l'Atlantique, prouvant - malgré les aveuglements linguistiques - une puissance dans les mutations et une logique des évolutions épistémologiques. Certes l'échantillon de géographes "américains» choisis pour ce colloque n'est pas représentatif d'une géographie "américaine» fermée sur ellemême : nombre d'auteurs sont d'origine européenne, alors que d'autres - francophones du Québec - sont bien au fait des évolutions de ces deux dernières décennies en Europe, qu'ils peuvent juger, critiquer en connaissance de cause.

Premier constat, celui de la nuance, apporté par V. Berdoulay: "Ce serait en effet déformer grossièrement la marche des idées que d'identifier une correspondance entre époque et paradigme ". Le cours de la pensée géographique reflète l'éclectisme de la géographie, sa diversité, malgré des interrogations existentielles lourdes : comment définir la nouvelle géographie? A-t-elle vraiment changé ? Est-elle désormais utile? Comment la pratique-t-on?

Deuxième constat, celui de la place de la géographie régionale. Même si la "proportion d'articles décrivant des lieux spécifiques " (A. Berubé) est tombée de $56 \%$ à $35 \%$ dans les Annals of the Association of American Geographers et de $80 \%$ à $57 \%$ dans les Annales de géographie, "l'objet régional» reste "une pierre angulaire de l'interprétation géographique " (A. Gilbert). Se posent alors les questions de l'organisation spatiale des processus sociaux associés au mode de production, suivant l'idéologie marxiste, bien représentée dans nombre d'articles nord-américains, et celles des espaces d'identification, sous-jacents dans les discours sur les lieux.

Troisième constat, celui de la dépossession graduelle « du mythe fondateur de la scientificité " (A. Berubé), chèrement acquis depuis 1950 (W. McTaggart). " Géographiser le social " (L. Deshaies) ?... Ce projet n'est-il pas en contradiction avec une discipline qui se veut à la fois «science pure et science appliquée ». Si les tenants « américains » de la pensée marxienne ne se posent pas la question, et font le projet géographique par l'analyse des enjeux territoriaux, des auteurs dont la pensée reflète d'autres idéologies s'interrogent sur la difficile utilisation du savoir géographique et de ses fondements scientifiques.

Au fait, pourquoi veut-on faire de la géographie une science? Pourquoi souhaitet-on définir son (ses) objectif(s) ? Pourquoi a-t-on peur qu'elle empiète sur les autres disciplines? N'est-elle pas avant tout un point de vue? Je crois que cette dernière question est la plus absente du questionnement fondamental du colloque, même si V. Berdoulay parle de désuétude du genre "poético-mythique", du récit de voyage, tout en nous rappelant les rééditions de textes anciens et les succès de Tristes tropiques. Cette question n'est-elle pas assez noble? Tout un courant de la géographie "américaine", peu représenté au colloque, traite de cette approche humaniste, qui ne se réduit pas à l'assimilation réductioniste paysage-langage de la sémiotique. Avec 
A. Berubé, nous écrivons que, si la géographie a perdu sa pertinence imaginaire et sociale, c'est qu'elle a trop souvent renoncé «à voir - et à faire voir - l'exotique dans le quotidien, l'anormalité dans le typique, le stupéfiant dans le normal ». II n'y a pas de géographie sans imaginaire, sans rêve... alors les lieux les plus humbles se parent de significations les plus profondes...

\section{Paul CLAVAL (Université de Paris IV)}

Jusqu'où convient-il de remonter dans le temps pour comprendre l'état actuel de la géographie ? Les collègues américains hésitent. Certains privilégient les perspectives courtes, comme Anne Gilbert ou Adrien Bérubé : ils ne sortent guère du présent. C'est également le cas de Juan-Luis Klein, de David Harvey, d'Allen Scott ou de Milton Santos, qui s'intéressent plus aux transformations du monde qu'à celles de la géographie. Plus nombreux sont ceux qui estiment nécessaire de partir des débuts de la géographie classique, à la fin du XIXe ou au début du XXe siècle. Vincent Berdoulay choisit enfin la perspective longue des types de discours que proposent les géographes.

La perspective moyenne, celle qui part des attitudes de la géographie classique pour mesurer le chemin parcouru depuis trois générations, nous est familière : elle met bien en valeur la part des héritages dans les démarches actuelles, tout en précisant ce qu'elles apportent de neuf.

L'optique de Berdoulay est plus étonnante: on l'avait connu privilégiant, dans ses analyses contextuelles, le rôle des modes intellectuelles dans la formation de la géographie classique. En s'intéressant aux discours, il s'installe dans la longue durée et paraît rompre avec ce qu'il avait fait jusqu'ici. Mais qu'apporte sa nouvelle approche? Le public attend de la géographie une présentation du monde, une certaine manière de le raconter, de le systématiser ou de l'expliquer et de comprendre ses évolutions. C'est à la fois montrer les forces de continuité à l'œuvre dans la discipline et la pesée du milieu dans lequel elle s'insère, puisque le discours attendu, ou accepté, oriente les curiosités. A travers sa contribution, Berdoulay nous offre donc une vision neuve des rapports entre contexte et disciplines scientifiques: l'idée de discours, si chère à Foucault, précise le rôle du milieu intellectuel. Berdoulay reste donc fidèle à ses préoccupations, mais les enrichit. En montrant la multiplicité des discours, si nette aujourd'hui, il fait comprendre la force vraie de la discipline : on attend d'elle beaucoup plus que ne le pensent communément les chercheurs.

Mettre davantage l'accent sur le dynamisme de la société que sur celui de la discipline paraît également original pour un Européen. Les fondements théoriques des contributions de Klein, de Harvey, de Scott ou de Santos ne nous sont pourtant pas inconnues, mais les thèses d'Aglietta sur la régulation et l'évolution du capitalisme vers des formes flexibles d'accumulation n'ont pas encore été exploitées de cette façon de ce côté de l'Atlantique. Cela dispense-t-il de se pencher sur le dynamisme interne de la discipline, sur la succession de ses interrogations? Olivier Soubeyran en doute: l'état de la géographie ne reflète pas seulement celui du monde qui nous entoure: la discipline est relativement autonome par rapport au cadre social et économique dans lequel elle se développe. 
Les nouveautés sur lesquelles insistent les textes «américains» ne sont pas toujours celles qui paraissent essentielles à la majorité des collègues continentaux. L'apport de l'écologie? II est certes jugé important ici, mais on l'a tellement associé à l'analyse systémique qu'on en a oublié les questions qu'il pose, et que W.D. McTaggart résume ainsi : "L'écologie profonde propose un «esprit» de la nature, semblable à l'esprit d'histoire de Hegel». C'est donc toute une philosophie qui est en jeu.

Pour Anne Gilbert, l'approche régionale est en train de se renouveler : sa modernisation a fait d'abord appel à tout un arsenal théorique qui devait permettre à la géographie de devenir applicable. L'orientation sociale qui s'impose aujourd'hui permet enfin de saisir la dimension concrètement vécue des problèmes. Cela devrait conduire à une réconciliation de la géographie et de la société: c'est là un point de vue rarement exposé en Europe.

La géographie des auteurs "américains"? Elle nous paraît très diverse malgré certains parallélismes dans les perspectives retenues. Une discipline malade? Certainement pas, à voir la diversité et l'originalité des points de vue qu'elle adopte - mais une discipline de plus en plus largement pratiquée par des non-géographes. De chaque côté de l'Atlantique, je crois qu'on est conscient de ce fait, fortement souligné par Laurent Deshaies.

\section{Daniel DORY (Université de Paris I)}

\section{Article de W. Donald McTaggart}

Une réaction épidermique tout d'abord : il me semblerait salutaire que nos collègues "américains " prennent vraiment conscience qu'ils ne sont pas les seuls habitants de la cité scientifique. Ceci conduirait peut-être à ne plus intituler « la géographie moderne... » un travail qui porte exclusivement (ou presque) sur la géographie actuelle aux U.S.A. (même si on la montre influencée par des auteurs issus des ténèbres extérieures).

Beaucoup plus fondamentalement, je suis en profond désaccord avec le renvoi dos à dos de la géographie « humaniste » et marxiste, qui négligerait également - bien que pour des motifs différents - les questions écologiques. Pour ce qui est de la géographie "humaniste", mélange hétéroclite de bricolage conceptuel et de contemplation du nombril, son intérêt pour le sujet qui nous occupe est trop mince pour qu'il vaille la peine de nous y attarder. Par contre, la problématique marxiste, nous paraît, elle, particulièrement appropriée pour poser les problèmes des rapports entre Nature et Sociétés. Et ce, au moins sous deux aspects. Premièrement, en ce qu'elle insiste sur l'historicité des termes en cause, Sociétés bien sûr, mais aussi Nature et enfin des rapports entre les unes et l'autre. En ce, depuis au moins l'Idéologie allemande (l'attribution à Engels dans La dialectique de la nature de la paternité de ce questionnement témoigne, d'ailleurs, d'une connaissance superficielle de la démarche marxiste, ce que confirment plusieurs passages du texte). Cette inclusion dans l'histoire de la nature elle-même permet de l'appréhender non comme mythe, mais partiellement comme condition et résultat de l'activité productive sociale, c'est-à-dire comme objet de connaissance et de travail. Ceci concerne directement la géographie et permet d'argumenter son projet scientifique à la fois naturaliste et sociologique de façon satisfaisante. 
Deuxièmement, en extrayant la problématique écologique de la seule sphère des idées - aussi généreuses soient-elles - pour la situer sur son vrai terrain: celui des politiques de l'environnement appliquées et/ou souhaitables dans chaque cas concret. Sur ce point également la géographie peut apporter une contribution éminente; mais elle dépend moins à ce stade de sa situation conjoncturelle présente que des besoins sociaux exprimés auxquels on la mettra en état de répondre, avec toutes les transformations que cela supposera tant dans son projet que dans son économie interne. Cela dit, je partage entièrement l'opinion de l'auteur concernant l'importance capitale des questions écologiques pour l'avenir immédiat et plus lointain.

\section{Article de Claude Pouliot}

Ce texte soulève un problème théorique passionnant, à savoir celui des conditions de scientificité d'un savoir exclusivement naturaliste pour autant qu'il s'inscrit dans le projet géographique. Je ne suis pas persuadé que la validité du discours géomorphologique puisse être conquise au moyen d'une rupture avec la nature, mais bien plutôt par sa réinsertion au sein d'une problématique d'ensemble - fondamentale - de la géographie. Alors la question de savoir comment la géomorphologie pourra être une approche scientifique naturaliste particulière et spécifique se posera autrement qu'en termes de simple mimétisme par rapport à la physique, la chimie ou tout autre domaine constitué.

\section{Article de Laurent Deshaies}

Je suis profondément d'accord avec le contenu de ce texte. En particulier les passages consacrés au territoire m'apparaissent d'une grande utilité. C'est, en effet, par le biais du concept de territoire que la géographie peut déployer sa double spécificité d'analyse naturaliste des sociétés et d'étude sociale de la nature. Le seul point sur lequel je ne suis pas l'auteur concerne le passage où il reprend la formulation - malheureuse, en l'occurrence - d'Henri Lefebvre. On ne peut dissocier, l'idéologique et la politique de l'ensemble des processus de «territorialisation ». Les sociétés produisent, en effet, simultanément des ignames et des rapports de parenté, du blé et des mythes, du mil et des dieux. Le territoire étant la condition et le produit de ce procès de production multiple et différencié, où les modalités du travail social sont déterminantes "en dernière instance". C'est ainsi, d'ailleurs, que l'on peut poser que la géographie est une science sociale, également "en dernière instance". Mais ceci est une remarque incidente par rapport à un texte que je n'hésiterai pas à qualifier de salutaire.

\section{Jean-Paul FERRIER (Université d'Aix-Marseille II)}

Onze textes reçus d'Outre-Atlantique - et le mien écrit il y a cinq mois. Douze textes donc, avant de nous rencontrer dans deux mois à Paris. Impression profonde d'unité, de responsabilité, de grande aptitude à contribuer collectivement à assurer les tâches qui attendent maintenant les géographes dans la communauté nationaleinternationale. Impression profonde. Les textes me sont apparus, dès leur première lecture, très convergents : la géographie doit bien en effet se préoccuper de la forme de ses énoncés, des conditions épistémologiques de son développement, de son apport à la construction de véritables modèles interprétatifs du monde d'aujourd'hui. 
Les géographes doivent en effet prendre mieux conscience de leurs places dans le système actuel de communication: dans un monde qui change, ils ont la charge de produire de grands récits dont on peut attendre la production de sens fondatrice de la culture vivante. A ce titre, il est certainement sage de ne se priver d'aucun des apports des géographes actuels, d'avoir ainsi une stratégie «multimédia » du discours géographique, visant alors des publics différents. Le repérage de "voies ou modalités de cheminement " (Orlando Peña) ou de "genres de discours " (Vincent Berdoulay) ou de "nouvelle géographie régionale" (Anne Gilbert) illustre cette entreprise. La confrontation avec les lieux et les régions est bien en effet le versant scientifique de la condition humaine de notre être-au-monde. On comprend que dans ce qui est inévitablement aussi une confrontation à une textualité, quand règne le "défi sémiologique", la géographie soit inséparable de formes nombreuses de géographie parallèle, et par conséquent qu'elle ne puisse se priver de géopoétique. Cette entreprise est de grande actualité - la nouvelle Géographie Universelle RECLUS l'illustre bien - si l'on accepte I'hypothèse que les sciences géographiques sont bien en phases avec les mouvements de la civilisation et peuvent contribuer à l'orienter. Le découpage que j'ai proposé, qui distingue trois modernités, guide nos "pas» et nous permet de mieux repérer les "destinations " souhaitables : la Troisième Modernité, porteuse de "reterritorialisation " garantit l'immense portée technique et culturelle - "sociétale» - de la géographie. Situation qui nous impose d'être mieux au fait de nos dynamiques épistémologiques et nous autorise à participer aux grands débats actuels sur le "prochain monde», la «sortie» de «crise».

Cinq textes se placent plus complètement dans le domaine épistémologique. Ils se partagent, ce qui me paraît significatif, entre une évaluation du contenu des discours (Adrien Bérubé, Olivier Soubeyran), une interrogation de notre rapport aux milieux "naturels» (Claude Pouliot, W. Donald McTaggart), une façon de situer la géographie dans le domaine des sciences sociales (Laurent Deshaies). Les revues institutionnelles (Annals of the Association of American Geographers et Annales de géographie) ou les pratiques professionnelles révèlent bien l' «état des lieux » : observons que la diminution de la géographie "régionale » sera compensée et au-delà par les textes actuellement en préparation autour de la Maison de la Géographie de Montpellier. Je crois à ce titre que les géographes ont repris leur "droit à la parole » sur les lieux et régions du monde, du territoire de la vie quotidienne au territoire-monde, et que l'explosion des moyens d'instrumentation de l'information géographique et d'édition multiplie nos possibilités professionnelles au moment où la demande «sociétale» redevient positive.

Mais cette prise de parole - reprise de parole - après le brouillage de nos messages territoriaux sous la Deuxième Modernité "déterritorialisante", n'aura sa pleine pertinence que si nous refondons clairement nos rapports avec l'interface nature-culture qui est au cœur de notre démarche : avec quels enjeux aussi bien pour la compréhension de nous-mêmes que pour celle du monde! La géomorphologie doit donc se renouveler épistémologiquement; elle doit être aux premiers rangs des courants de l'écologie "profonde». Pour ces raisons, je souhaite mettre en discussion à Paris la conception de la géographie comme science sociale ou comme science de l'homme et de la société. En effet, si l'on accepte l'hypothèse d'une double tendance forte à la montée de l'individuation de la personne et à la mondialisation, n'allons-nous pas être embarrassés par les notions des sciences sociales développées pour l'essentiel sous la Deuxième Modernité dont les procédures étaient si contraires à l'approche du territoire? Des conceptions trop restrictives peuvent nous empêcher d'aller au bout de notre projet. 
Last, but not the least, trois textes (Milton Santos, Juan-Luis Klein, David Harvey et Allen Scott) placent la géographie face aux "enjeux territoriaux dans la société de l'après-crise ". Enfin! Car nous avons été bien silencieux et bien suivistes (le géographe, ou la voix de son maître) pendant la Deuxième Modernité, à la remorque de l'économie ou des discours technocratiques, souvent après édulcoration journalistique. Sort dont nous comprenons maintenant seulement qu'il était difficile à éviter quand la science des territoires était confrontée à la "déterritorialisation» du monde. Or, dans la Troisième Modernité, "on dirait (...) qu'il n'y a que deux choses : capital financier et géographie, c'est-à-dire son réinvestissement dans un lieu " (Milton Santos). Dès que nous partageons ce point de vue - en ajoutant qu'il y a les hommes et leur pleine culture, part irremplaçable aussi de la « ressource humaine» - la géographie retrouve une pertinence perdue, la retrouve plus grande qu'auparavant à cause de l'explosion technologique et communicationnelle: elle est partie prenante des grands systèmes interprétatifs et évaluatifs du monde en gestation, elle est beaucoup plus que géopolitique, elle est fondamentalement géostratégique. En soumettant à la discussion qu'il n'y aura pas de géostratégie porteuse de paix et d'humanisation du monde sans géopoétique. Carl'homme « ressource » dans les lieux « ressources » doit suffisamment réussir sa vie, corriger les inégalités, réguler la solidarité du local au global, habiter... Cet homme, chacun des habitants de la planète Terre, ne mérite-t-il pas d'habiter " poétiquement " (Hölderlin)?

Dès lors nos façons de nommer le monde et ses grands mécanismes sont nécessaires et doivent être justement évalués. Ne faut-il pas justement commencer par nous imposer de désigner la machine «sociétale » et le système-monde en employant des notions et des concepts développés dans un univers théorique qui nous soit propre, qui soit fondamentalement intragéographique? Pour éviter, par doute méthodologique, de reprendre les catégories de la Deuxième Modernité, quelles soient celles de l'économie ou celles de la technologie. Pour nous imposer de "géographier» les outils et les mécanismes. Pour construire dans un système épistémologiquement plus clos où nos mesures sont des mesures humano-centrées, où la question du sens comme celle du bien et du beau est recevable.

\section{Yves GUERMOND (Université de Rouen)}

Les textes de Harvey et Scott ont cette énorme qualité de ne jamais laisser indifférent. Ils sont pleins d'idées et me laissent toujours admiratif devant la qualité de la démonstration, en même temps que perplexe sur le fond. Puisque ce débat m'en donne l'occasion, je vais essayer d'expliquer pourquoi.

Je commencerai par indiquer de larges points d'accord avec ce qui est dit par les deux auteurs, afin de restreindre le débat à ce qui peut être objet de discussion. Je suis d'accord (qui ne le serait?) sur l'idée de l'émergence actuelle de secteurs de production totalement nouveaux, et sur le rythme accéléré de l'innovation technique et commerciale. De même, il paraît évident que ce sont des mécanismes de nature capitaliste qui dominent l'organisation des loisirs, la production des connaissances (du moins leur diffusion), la division du travail au sein des ménages (encore que ce soit moins certain), etc. On doit admettre aussi que les formes d'accumulation, dans les sociétés capitalistes 
avancées, sont moins dépendantes qu'autrefois de la production de masse fordiste. Enfin, ce n'est pas moi qui vais nier que "nous sommes conduits à centrer notre attention plus spécifiquement sur le rôle de l'espace, dans la compréhension des processus de l'accumulation flexible».

A côté de ces quelques évidences, les auteurs cherchent à élargir parmi leurs lecteurs le champ du consensus, en utilisant une technique bien particulière qui consiste à prendre position catégoriquement, ici ou là, contre des idées qui n'ont jamais été émises sérieusement par personne: «nous nous opposons à la notion selon laquelle la phase actuelle du capitalisme serait marquée par de fortes tendances désorganisatrices, dans lesquelles la cohérence et l'unité du système capitaliste se dissoudrait ». Qui prétend celà? Un peu plus loin, les auteurs s'opposent aussi à la notion selon laquelle "l'histoire et la géographie seraient construites par les êtres humains dans des lieux uniques ayant un avenir ouvert ", et ils rejettent «l'idée qu'il y ait quelque opposition que ce soit entre la théorie et l'observation contingente, dans l'analyse matérialiste historique ". Heureusement tout de même, à moins qu'on trouve normal de dire n'importe quoi. On peut ne pas être d'accord avec Marx, mais il faut reconnaître qu'il part toujours d'exemples empiriques très précis. C'est ce qu'il appelle le procédé d'investigation. On n'en parlerait plus aujourd'hui s'il avait agi autrement.

Le cœur de l'exposé est constitué par le concept «d'accumulation flexible », qu'on nous demande d'admettre pour remplacer le concept précédemment admis par les milieux post-marxistes de «capitalisme monopoliste d'État». Ce concept précédent reposait sur l'idée, maintenant abandonnée, " qu'un certain mouvement vers le socialisme pouvait être discerné dans le développement d'un capitalisme de transition ». Rien ne permet d'affirmer que les nouvelles thèses offrent davantage de sécurité que les anciennes, et on tourne en rond dans le débat théologique pour initiés et convaincus.

L'accumulation flexible n'est pas géographiquement aussi évidente qu'on le dit. Certes des usines japonaises s'installent un peu partout, mais seules les usines de montage sont "flexibles", comme l'a montré Benoit Brault (L'Espace géographique, $1987, n^{\circ} 3$ ) à propos de celles qui se sont installées en France. Chacun connaît les efforts sans succès de Singapour pour se doter de véritables entreprises de High Tech. Les auteurs croient discerner une accentuation du mouvement des capitaux industriels hors des vieilles régions de production vers divers sunbelts, et c'est là un thème très à la mode. C'est vrai si l'on observe les pourcentages d'accroissement, par rapport à des niveaux d'industrialisation très faibles au départ, mais en termes d'emplois, l'héritage spatial demeure.

Il est toutefois inévitable que la polarisation industrielle et urbaine sur le charbon et l'acier, qui n'a plus de justification technique, ne se maintienne pas éternellement, alors qu'on peut localiser ailleurs l'industrie des matières plastiques, l'électronique, et les industries spatiales. II n'est pas étonnant non plus que des régions de croissance spectaculaire se trouvent brutalement abandonnées : c'est ce qui se produit à chaque fois que les facteurs géographiques de localisation sont circonstanciels: l'actuel développement de Larnaca est lié à l'occupation du Liban par la Syrie et Israël, mais si le Liban retrouvait sa liberté, les conditions locales ne favoriseraient plus l'île de Chypre. Si l'on retire du débat ces trois types de déplacements des activités (l'illusion statistique des sunbelts, les causes techniques liées aux sources d'énergie, les causes politiques circonstancielles), "l'instabilité profonde » évoquée par les auteurs à l'appui de la thèse de la "flexibilité » est ramenée à de plus justes proportions. 
Il faut éviter les modes dans toute tentative de théorisation. C'est ainsi qu'on ne peut admettre l'idée émise d'un «mouvement quasiuniversel vers une politique néoconservatrice». C'est Marx lui-même qui disait qu'on «ne juge pas une époque d'après la conscience qu'elle a d'elle-même. La structure économique de la société (est) la fondation réelle sur laquelle s'élève un édifice juridique et politique " (Avant-propos de la Critique de l'économie politique). L'épisode politique du «libéralisme» des années 80 ne se situe pas au même niveau explicatif que les transformations réelles du système productif, et il ne faut pas accorder une aussi grande importance que le font les auteurs à la situation momentanée de la vie politique des USA et du Royaume-Uni ces dernières années. Aux USA, l'arrivée de Reagan au pouvoir a été largement due à la révolution iranienne, et on connaît peu de gouvernements qui, confrontés aux difficultés rencontrées par Carter, auraient pu s'en sortir. Au Royaume-Uni, l'arrivée et le maintien du gouvernement conservateur doivent beaucoup à la stupide division de l'ancien Labour Party mais, ni dans un cas ni dans l'autre, on ne peut affirmer qu'il s'agisse d'une tendance lourde au conservatisme. Les auteurs sont abusés ici par leur perspective anglo-américaine. En France, François Mitterrand vient d'être réélu avec $54 \%$ des voix. Les auteurs se trouvent pris eux-mêmes au piège de la "glorification de l'esprit d'entreprise ", qu'ils dénoncent pourtant d'une part mais qu'ils considèrent d'autre part (la "compétence des entrepreneurs») parmi les "contraintes géographiques», au même titre que l'environnement physique et que les structures préexistantes du développement urbain et régional. On sait bien pourtant que la compétence des individus est uniformément répartie sur la planète, au hasard des individus, et que seules changent les conditions géographiques qui permettent ou ralentissent son épanouissement.

Tout le problème est de déterminer la part d'explication qu'il convient d'imputer au mode de production dans la société actuelle. On peut raisonnablement se demander si "la compression espace-temps" est bien, comme on l'affirme spécifique du "monde capitaliste "... L'affirmation est pourtant répétée plus loin dans le texte : «l'annihilation de l'espace par le temps peut être considérée comme essentielle à la dynamique du mode de production capitaliste». Comment affirmer de même que "le glissement massif de l'emploi vers le secteur des services" soit le résultat d'une évolution propre aux pays capitalistes ? Si l'on veut analyser les effets du capitalisme, il n'y a pas d'autre manière scientifique de le faire que d'isoler ces effets de ceux des autres variables. Le degré de pénétration d'une économie par le capitalisme est une variable comme une autre, elle n'a pas de statut particulier, sauf dans un discours de type théologique. On peut sourire également du "contexte de diminution de la productivité", qui est présenté comme une caractéristique de notre époque. Tout le monde peut donner des exemples, dans un certain nombre de branches (l'informatique, la chimie fine, les cosmétiques) d'accroissements considérables de la productivité... sans que le capital ait changé de place.

D'autres affirmations reposent sur des bases incertaines: le changement «dans des proportions monumentales » de la logique de la géographie capitaliste est attribué "à des attitudes culturelles post-modernes", ce qui est relativement flou. La "monétarisation de la vie quotidienne", est contredite par de nombreux auteurs, par exemple A. Frémont (France : Géographie d'une société, 1988) : «l'observation empirique révèle une très grande densité d'échanges, qui concernent particulièrement la garde des enfants, l'aménagement de l'habitation, des petits services très variés, les ressources alimentaires en provenance de la campagne... Les systèmes de relations informelles et le tissu associatif concourent partout, avec la fréquentation usuelle des commerces et des services, à la cohérence des rapports de localité ». Le tissu social existe, quel que 
soit le mode de production ambiant. Si un rôle peut être fixé au géographe, c'est peutêtre de contribuer à renforcer ce tissu social. Ainsi, à propos du "local», évoqué par Scott et Harvey, le problème n'est nullement de savoir s'il s'agit d'un concept " puissant " ou non. Les géographes qui travaillent sur «le local » le font en le considérant comme une volonté à laquelle on doit faire prendre corps : « le dynamisme individuel et collectif autour d'un projet de développement culturel... répond d'abord à un besoin intérieur à la communauté, celui de la rencontre et des relations intergénérations entre habitants » (N. Mathieu : inerview au Quotidien du Maire).

Deux choses sont intéressantes dans la vie d'un universitaire: la recherche scientifique méticuleuse de l'enchaînement des causes des phénomènes, d'une part, et d'autre part la contribution au renforcement du tissu social. Dans ces deux domaines, d'infinies perspectives d'action s'ouvrent au géographe, en tant que géographe, mais à condition de ne pas se racornir sur une spéculation intellectuelle fonctionnant pour elle-même en auto-alimentation.

On ne voit pas bien ce que le savoir géographique peut apporter à la solution des problèmes de la monnaie, qui sont évoqués dans le texte. Globalement, la structure théorique proposée demeure sans grand effet pratique sur la géographie, car elle ne parvient toujours pas à faire glisser le marxisme de ses bases historiques pour l'amener à correspondre à des bases spatialisées. L'objectif affirmé des auteurs est d'ailleurs défini comme un désir d'interpréter «la géographie historique » du capitalisme, c'està-dire, en clair, son "histoire».

II ne me semble pas non plus qu'on puisse dire que la théorie proposée puisse conduire à " un changement social émancipateur ». Les grands enjeux sociaux actuels me semblent être d'un côté la constitution au sein des pays développés d'une société à deux vitesses, avec un secteur perpétuellement assisté et dépendant, et d'un autre côté l'appauvrissement constant du Tiers-Monde. En bref, ce qui me motive vraiment, c'est d'espérer qu'on pourra un jour, en partie grâce à nous, et pour reprendre la formule de Pierre Teilhard de Chardin, mettre fin "à la scandaleuse et chaotique dispersion humaine". Je n'arrive pas à voir en quoi la compréhension des origines et de l'impact de l'incomplète transition vers l'accumulation flexible, qu'il nous est demandé " d'accepter collectivement " (sic) comme objectif en tant que géographes, puisse guider mon action personnelle, ni comme chercheur scientifique ni comme homme, car il s'agit d'une préoccupation d'histoire économique et non de prospective sociale.

\section{Christian KESTELOOT (Katholieke Universiteit te Leuven)}

La première impression qui se dégage d'un rapide survol des textes "américains ", est que la plupart d'entre eux sont canadiens ou en tous cas, qu'ils se situent dans la mouvance francophone de la géographie (à l'exception des textes de Harvey et Scott et de Mc Taggart). L'interaction visée me semble porter d'une part sur les différences de développement existant entre l'Amérique du Nord francophone et l'Europe et, d'autre part, sur les nouveaux thèmes géographiques que ces développements appellent; et cela bien qu'une confrontation entre les mondes géographiques anglo-saxon et francophone m'aurait paru plus enrichissante (les clivages linguistiques formant une barrière non négligeable à la confrontation des idées). 
La seconde impression a trait aux textes qui, de façon explicite ou implicite, font référence à l'approche de l'École française de régulation, ou plus généralement d'une théorisation des développements du capitalisme qui tienne compte à la fois des crises économiques et des restructurations du capitalisme qu'elles réalisent. En découle une périodisation de la croissance économique où chaque phase connaît une structuration propre de l'économique, du social et du politique qui caractérise le mode de croissance. L'importance de ces phénomènes pour la géographie vient du fait que cette structuration se réalise à travers une logique caractéristique de l'organisation de l'espace et que l'on peut retracer les limites de ces modes de croissance dans les freins que lui oppose l'organisation même de l'espace (de par son inertie dans le temps et la "lourdeur " de ses structures spatiales par rapport à la nécessité d'accélérer les activités de production, de distribution et de consommation).

Ce diagnostic soulignant l'importance du spatial semble assez bien accepté chez les auteurs qui s'appuient sur une analyse de la crise et du développement éconornique pour expliquer les nouveaux enjeux de la géographie et interpréter les changements épistémologiques de ces dix dernières années. Mais je suis également frappé par le caractère quasi anecdotique des références aux concepts de l'École de régulation utilisés pour y parvenir. Bien qu'il y ait chez plusieurs géographes "américains " un consensus pour adhérer au même corps d'idées, ces références ne sont pas assez rigoureuses pour que l'on puisse parler d'un tronc théorique commun. Milton Santos, par exemple, nous livre un papier qui traite très clairement du thème, mais les termes utilisés sont tellement généraux qu'il aurait tout aussi bien pu l'avoir écrit il y a un siècle pour traiter des problèmes qui se posaient à l'époque. Klein nous fournit une étude où il fait explicitement référence à la théorie de la régulation, mais où il semble méconnaître des pans entiers de cette approche (pas spécialement ceux qui ont été les plus développés par les économistes de l'École de régulation, dont les références à l'organisation de l'espace, à l'instar de Marx, se résument à quelques phrases assez cryptiques - à l'exception de Lipietz peut-être qui accorde beaucoup d'importance à l'organisation de l'espace capitaliste à l'échelle mondiale). Néanmoins, il me semble que Klein réduit l'utilisation de cette approche à l'interprétation d'un phénomène tellement marginal de la restructuration de l'organisation de l'espace (les relations entre les communautés spatiales et les pouvoirs publics locaux), qu'il se prive des moyens d'expliquer d'une façon plus globale les enjeux dont il traite. Le texte de Harvey et Scott m'est par contre apparu beaucoup plus proche de la problématique qui nous était soumise et m'a semblé beaucoup plus riche et rigoureux sur le plan conceptuel pour comprendre les enjeux de la géographie dans le cadre des réorganisations spatiales qui découlent de la crise et des stratégies pour en sortir.

Je tire de ces comparaisons l'enseignement qu'il est urgent - peut-être de concert avec les économistes et d'autres chercheurs en sciences sociales qui s'intéressent à la dimension spatiale des phénomènes sociaux — de définir clairement un tronc commun de concepts et de relations abstraites qui permettent de mieux analyser la réalité géographique. Je crois que les idées développées au sein de l'École de régulation nous permettent de faire un important pas en avant. Mais au préalable, il nous faut maîtriser l'ensemble de ces concepts, pour éviter d'en faire une utilisation ad hoc, trop partielle pour convaincre de la nécessité d'établir une géographie vraiment sociale qui ait trait aux dimensions et enjeux spatiaux de la société.

Les autres textes, sauf celui de Pouliot, avalisent tous d'une façon ou d'une autre le « retour du local » et l'affirmation de la géographie humaine en tant que science sociale. Mais aucun ne précise les causes de ces changements par une analyse de l'évolution sociale et économique et de son influence sur la géographie. En d'autres termes, ils ont 
tendance à attribuer à la géographie un développement autonome par rapport à la société. Ils ne reconnaissent pas la production scientifique comme une pratique sociale sous l'angle des demandes sociales et de leur évolution. Tout au plus font-ils un rapport entre la géographie et la planification spatiale, ce qui revient à voir cette pratique sociale sous l'angle de l'offre de connaissances et de techniques de gestion de l'espace. On part donc de l'idée que la géographie peut influencer la société de façon autonome, et la plupart des textes cherchent à trouver le moyen le plus efficace d'atteindre cet objectif - que ce soit au niveau du discours utilisé (Berdoulay), des piliers à unifier (Peña), du redéveloppement de la géographie régionale (Gilbert), de la promotion du concept de territoire (Deshaies) ou d'une meilleure conscience de ce qui intéresse le grand public (Bérubé).

Deux textes font exception: celui de Mc Taggart et celui de Soubeyran. Je reviendrai au premier plus loin. Une première lecture du texte de Soubeyran m'a donné l'impression que je présente de façon trop déterministe l'influence de la demande sociale sur l'évolution de la géographie, et que les relations entre les développements de la géographie et ceux de la société sont bien plus complexes qu'il n'y paraît à première vue. II y a des marges de manœuvre, des champs d'autonomie relative, des interties du passé qui permettent à la géographie de suivre d'autres cheminements que ceux qui lui seraient imposés par l'évolution de la société. Mais les constats de Soubeyran ne semblent pouvoir se faire qu'a posteriori. Il illustre ses propos par une analyse de la géographie française classique et se limite, pour la géographie actuelle, au problème de la planification (alors que le renouveau d'intérêt général pour l'espace n'est certainement pas lié au seul fait qu'on puisse en planifier le développement). J'aimerais connaître la façon dont Soubeyran appliquerait ses concepts aux enjeux de la géographie actuelle, tels qu'ils sont présentés par Harvey et Scott, Klein et les géographes européens qui travaillent à partir d'une même analyse de l'évolution des sociétés et de leurs enjeux spatiaux.

A mes yeux, c'est le texte de Mc Taggart qui suscite le plus de réactions immédiates: il est à la fois le plus immédiat au niveau du diagnostic (à l'exception du texte de Harvey et Scott avec lequel je me sens tout à fait d'accord) et le plus lointain dans ses projets. Les problèmes écologiques sont fondamentaux et il est vrai que les géographes (du moins s'ils maîtrisent à la fois les sciences naturelles et sociales, ce qui n'est pas toujours évident) peuvent réfléchir sur ces problèmes. Je ne suis cependant plus d'accord avec lui lorsqu'il présente ces problèmes comme indépendants des structures sociales et économiques. Devra-t-on vivre sous le totalitarisme de la nature (l' «esprit de la nature» à l'instar de l' "esprit de l'histoire" de Hegel) pour échapper aux catastrophes écologiques? Qui décide de cet "esprit de la nature»? Qui peut le connaître ? Quel pouvoir cela lui donne-t-il par rapport à ceux qui n'ont pas les moyens de le connaître? Nous voilà repartis pour une société féodale où l'Église serait remplacée par les géographes écologistes. Que les mêmes problèmes se posent dans les pays socialistes n'enlève rien au fait que, chez nous, c'est le capitalisme avec son besoin constant d'accumulation et de croissance économique qui provoque ces problèmes et empêche à la fois de les solutionner. Je ne réfute pas ici l'idée qu'il est important d'étudier les problèmes écologiques, bien au contraire. Mais il faut réfléchir sur l'approche qu'on utilise dans ce cas, pour tenir compte des effets de pouvoir que l'on provoque. McTaggart semble d'ailleurs ignorer que beaucoup de ces problèmes sont étudiés sans qu'il soit nécessaire pour cela de faire profession de foi envers "l'esprit de la nature» (autant les géographes traditionnels, théoriques et quantitatifs que marxistes ont étudié et étudient les problèmes de la faim dans le monde, du 
déboisement, des nuisances industrielles, de l'épuisement des ressources naturelles, etc.).

La lecture de l'ensemble des textes «américains (je n'ai pas lu les textes européens, mais je présume que les mêmes tendances peuvent s'y discerner) m'amène à une interrogation que je trouve inquiétante : les géographes adhèrent-ils au projet scientifique qui est de participer à l'émancipation des hommes (et des femmes) par la connaissance? Ce projt, du moins pour les géographes belges qui l'ont adopté, est devenu explicite grâce aux géographies théorique et quantitative qui dans, un premier temps, leur ont révélé la possibilité de contribuer à l'amélioration de la société par une étude systérnatique de certaines facettes de l'organisation sociale de l'espace, ouvrant ainsi des perspectives normatives. Dans un second temps, ils ont compris que cet aspect normatif n'était rendu possible dans le courant théorique et quantitatif que par une approche trop abstraite de la réalité, ignorant les enjeux, les rapports de pouvoir et les intérêts différenciés des groupes sociaux dans une société fondamentalement conflictuelle. D'où l'adoption ultérieure de concepts marxistes et/ou weberiens. Aujourd'hui, ce type d'approche est suffisamment développé pour que d'autres géographes puissent à la fois y puiser des idées qui n'impliquent plus l'adhésion à la démarche radicale de ses initiateurs, et pour qu'ils puissent mettre le doigt sur les erreurs, des exagérations ou des caricatures évitant ainsi que soit discréditée la démarche dans son ensemble.

A mon avis, cette démarche radicale est, en tant qu'émanation de la révolution théorique et quantitative de la discipline, le mouvement le plus développé actuellement en géographie en regard du projet scientifique défini plus haut. La dilution de cette approche radicale dans le paysage épistémologique (tel qu'il apparaît dans les textes présentés ici), la sensibilité que les géographes portent aujourd'hui au « marketing » de leur produit, et l'absence de réflexion (à part le texte explicite de Harvey et Scott) sur les relations actuelles entre les développements sociaux et la production scientifique géographique, me font penser que ce projet scientifique n'est pas partagé par l'ensemble de la communauté des géographes, ou qu'il se perd à force de rechercher une pertinence sociale trop immédiate. À mes yeux, il n'y a cependant qu'un seul domaine dans lequel il vaille la peine de créer l'unité de la géographie : c'est celui de joindre ses efforts pour contribuer à l'émancipation des êtres humains.

\section{Eleonore KOFMAN (Londres)}

Several of the "American" contributors focussed primarily on the nature of economic, social and political change and its territorial implications under contemporary capitalism. The fact that this form of questioning found greater expression in the "American" that the European texts in the colloquium is probably due to the state of geography in the respective societies as well as the choice of geographers. My own comments will be limited to the three papers which fall into the category I have mentioned above Harvey and Scott, Klein and Santos, although McTaggart also broaches a major contemporary debate which should engage geographers, that of ecological destruction and equilibrium. Having said that I will concentrate on three papers, I will, nevertheless pay much less attention to Santos who, after suggesting that the rapidity of change requires new definitions and methods of analysis, then procedes no further than an outline of the new modes of production in an era of economic concentration and the 
increasing deployment of scientific and technical knowledge in production. What seemed to be the most interesting questions arising from this restricted set of contributions was the what sort of theory and its appropriate level of theorisation necessary to understand our changing world, and what role geographical knowledge has to play in this process. Despite the various disclaimers, especially from Harvey and Scott, the questions seemed to be posed unproblematically.

\section{Rationality Rules OK}

All three papers believe in a rationale analysis of the current transformation from fordism to flexible accumulation and its corresponding form of regulation. For Harvey and Scott, this troubled period of readjustment on all fronts provoked deep cleavages in Marxist theory and more generally a theoretical crisis in the late 1970s, including a fixation on the specificity of the local. However, they argue that we should not be led to believe that "the coherence and unity of the capitalist system is dissolving away"; we must penetrate instead beyond the surface appearance of the fragmentary, the contingent and the ephemeral and elucidate the generality of capitalism in its totality so as to produce a holistic theory. This involves a "project of understanding the totalizing behaviours of capitalism." Whilst I agree entirely with the rejection of the supposed disorder proclaimed by much post-modernist theorising and the retreat into unconnected locality studies, I fear that the logic of accumulation is still expected to explain all processes and practices. I shall return to a critique of a single form of regulation which is shared by Klein, but for whom one of the key elements of the new post-keynesian form of regulation is territory, since it is at the local and regional levels that coherence and cohesion is produced now that the state has run into difficulties in doing so. It is here that a new social consensus can apparently by soldered (Klein). Klein's is a richer, less linear theorisation, fully expressed in his concluding remarks on the value of "une approche, à la fois, systémique et dialectique, selon laquelle l'étude des lieux doit tenir compte de l'appartenance de ceux-ci à une totalité, mais aussi de leur autonomie et de leur capacité d'influencer cette totalité".

What then is the relationship between the different dimensions of change, of various territories and scales, of the individual to the society of which she is a member. Given that these are all enormous questions, I don't propose to enter into all the issues but simply to point out a few rather pertinent aspects.

There is no single, hegemonic regime of capitalist accumulation nor model of development at present (Leborgne and Lipietz, 1988, L'après-fordisme et son espace. Les Temps modernes, 43, 501: 75-114). Forms of flexibility and its importance vary nationally as well as regionally from the neo-taylorian (polarised development and highly segmented labour markets, increasing poverty), Californian (individual negotiation) and the Saturnian (individual combined with collective negotiation over salaries and conditions of work based on a certain level of social consensus and rejection of a dual society). What is significant is that the dominance of one or other model at a particular period in a particular territory is not just the result of the pure logic of capital; it arises from socio-political acquiesence, consensus or conflict. It would be interesting to examine what situations have led to a strongly territorial mode of regulation, a model that certainly dosen't apply to Britain where even the relative autonomy required to initiate local social concertation has been squashed. In this case territorial regulation would present an unacceptable obstacle to flexibility.

However, apart from the existence of multiple forms of regulation, the attempt to construct a totalising theoretical framework, despite affirmations to the contrary, tends 
to assume that all geographical and other aspects of change result from the consequences of a logic of transformation. It is easy to read a logical relationship into what seems to be a coherent and systematic whole, and ignoring the partially autonomous origins of the parts. If we stick to the example of the role of territory in the current system of regulation (leaving aside how many there are), it all appears to have emerged out of the disengagement of the State. Yet, in France at least, early initiatives at autonomous, local development and the modern discourse focussing on the pays can be traced back to the 1960s in Britanny (Quéré, 1981 Régions et pays en Bretagne. Hérodote, 23: 70-98). In more socially mobilising version, "vivre et travailler au pays" was popularised by the Occitan movement, and about the same time in the early to mid-1970s, recuperated by the State and the Left, each with their different emphases. Other social movements were also subsequently partially incorporated into a coherent whole, but it is wrong to reducce their significance and meaning to the transformation of a system of regulation.

\section{The Places of Emancipation}

The importance of sound theory is that it provides us with the "construction of coherent representations and analytical tools to facilitate the struggle for human emancipation" (Harvey and Scott). Obviously the use of theory for this purpose is not shared by all geographers (see commentary by Guermond), but my own comments are derived from a critical stance of the relationship between theory, the researcher and the society in which the latter is involved. Yet, it is all too easy to assume that because we have the correct theoretical foundation, that it facilitates emancipation. Are our theoretical jousts merely destined to circulate among those already initiated? We need to give honest answers to questions concerning for whom our knowledge is intended, how it is used and its actual effectiveness in creating the conditions for social and political change.

It would be a pity if we let ourselves become trapped in a debate polarised around a search for a totalising theory and those more convinced of the value of empirical research - admittedly a highly simplified conceptualisation. The tendency amongst those developing a totalising theory is to conceive of emancipatory efforts in terms of the logic of this transformation. Yet, I would strongly dispute that we can align the various dimensions of discrimination, exclusion, subordination, etc. to this logic of regulation. For example, women's subordination and oppression, whether in North America, Europe or Africa, cannot be neatly packaged in these terms. This dosen't mean in turn that we should devote all our attention to developing a more sophisticated totalising theory which fuses capitalism and patriarchy. It is important not to close off all that dosen't fit easily into this conceptualisation and to remember that one of the most significant contributions of feminist theory has been to stress the diversity of forms (both overt and subtle) and places in which women are discriminated, oppressed or excluded.

\section{Jean-Bernard RACINE (Université de Lausanne)}

Ayant choisi moi-même de répondre, du côté européen, au questionnement des organisateurs du débat transatlantique Géographie, état des lieux en posant au départ que l'on pouvait organiser la réflexion critique sur quelques thèmes essentiels renvoyant 
tous à la question fondamentale de la place et de la pertinence du référentiel théorique qui encadre, explicitement ou non, les connaissances et les pratiques que les sociétés ont de leur espace, c'est également de ce point de vue que j'ai choisi de lire les différents textes proposés par mes collègues d'outre-atlantique. Mais pouvait-on en rester à ma propre vision des trois composantes essentielles de ce référentiel théorique, qui renvoyaient pour moi, au modèle de l'homme, au modèle d'espace et finalement aux représentations que le géographe s'est forgées ou qu'a successivement ou aternativement empruntées, à titre de référent, la pratique géographique?

Apparemment oui, en tout cas pour qui commencerait la lecture des textes "américains " par celui de David Harvey et Allen Scott. Ces deux chercheurs ont, entre autres, en commun d'avoir un projet explicite (a geographical agenda) pour la géographie, projet dont on s'étonnera un peu qu'on le retrouve ici sans autre élaboration originale que la présentation d'une hypothèse de recherche valorisant l'importance d'un espace de la force de travail hautement différencié dans ses qualités et ses configurations, comme conditionnant majeur de la mobilité géographique et de la flexibilité de la production du capital. Cette hypothèse n'est pas nouvelle et sa pertinence, qui n'est d'ailleurs que partielle, semble avoir été largement évaluée. Pour le reste, il n'y a pas grand chose de neuf à dire quant à la prise de position de nos deux collègues: le référentiel marxiste comme toile de fond nécessaire à la pratique de la géographie humaine dans le cadre de la prise en compte de la transformation actuelle du procès d'accumulation capitaliste est certainement l'un des passages obligés de l'intelligence de la production des formes spatiales et des modes de vie et de relations qui s'y inscrivent tout en contribuant à leur transformation. Reste à savoir si c'est le seul, s'il est suffisant, et si nos collègues ne pèchent pas à leur tour par réductionnisme, une vision holistique du monde n'étant pas exclusive, entre autres, de la prise en compte du problème des formes d'existence historique de l'individualité. On sent les auteurs mal à l'aise envers tout ce qui rappellerait, de près ou de loin, le paradigme weberien et l'interprétation qu'en donne un Raymond Boudon par exemple. Voulant à tout prix faire du rapport d'exploitation (et plus spécifiquement peut-être des «lois » du mouvement d'accumulation du capital), qu'il ne faut certes jamais oublier, l'élément central de la description et de l'explication, nos deux auteurs illustrent bien involontairement les effets non intentionnels de la problématique, du même nom, qu'ils dénoncent : être à leur tour dénoncés comme réductionniste et réificateurs.

Quitte à travailler sur des "abstractions concrètes", autant se donner comme ambition de ne pas réduire ces dernières aux seules composantes, certes évidentes, des configurations géographiques de l'économie spatiale et chercher les outils d'intelligibilité d'une géographie du monde plus complexe, osant mettre en perspective réciproque le subjectif et l'objectif, le psychique et le comportement, l'aménagement et l'usage, le spatial et le social, dans une visée plus authentiquement transactionnelle. Et ce d'autant plus que l'on a l'ambition, explicite chez ces auteurs, de contribuer à la prise de conscience politique, par les intéressés eux-mêmes, des enjeux sociaux des transformations en cours. Comment ne pas s'étonner à cet égard de la grande ambiguïté du projet politique évoqué, pour ne pas dire de sa vacuité dans la manière du moins dont ils évitent de lui donner le moindre contenu précis?

Heureusement, les lecteurs connaissent suffisamment l'œuvre par ailleurs magistrale, de David Harvey et Allen Scott, pour ne pas en juger définitivement sur la simple présentation de ce texte dont l'utilité marginale reste à démontrer. La grande réussite du récent essai de liaison entre accumulation flexible et post-modernisme dans la cité que David Harvey nous a proposé dans la revue Antipode, comme tant d'autres travaux 
d'Allen Scott aussi, nous confirme cependant dans notre conviction que ces deux maîtres de la géographie moderne continueront à jouer un rôle de premier plan dans le «remodelage de la géographie». Je les en remercie d'avance.

Les autres articles, dont certains s'inscrivent assez directement dans la ligne des prises de position « d'amont » de Harvey et Scott d'ailleurs, tel celui de Juan-Luis Klein, viennent heureusement compléter leur propos en l'élargissant et en illustrant en particulier le rôle nouveau du territoire et du sentiment identitaire dans le développement local, sans rien renier de ce qui relève des contradictions sociales les plus fondamentales, au sens premier du mot. Dans sa facture d'ensemble comme à travers l'exemple proposé, le travail de Juan-Luis Klein m'apparaît comme particulièrement pertinent. L'apport de W.D. McTaggart est également significatif du nécessaire dépassement de problématique marxienne à usage interne. C'est dans une perspective un peu analogue que j'avais personnellement plaidé pour la mobilisation, dans l'étude du rapport de I'homme total au milieu total, d'un concept «bio-culturel» de l'homme, que W.D. McTaggart a su expliciter dans une perspective tout à la fois plus riche et plus directement géographique, en en montrant heureusement sa pertinence sociale très actuelle.

Pertinence évidente aussi dans l'apport des réflexions que nous offre Milton Santos sur les règles des temps nouveaux. A ce niveau de généralisation il est bien difficile de ne pas être d'accord avec l'auteur et il était important de souligner le rôle des changements technologiques encore que l'on eût pu souhaiter que l'auteur aille plus avant dans son analyse des effets des mouvements de la structure globale sur chaque lieu, les lieux étant qualifiés par des populations concrètes, dans leurs structures, leurs connaissances et leurs pratiques de l'espace.

Restent les textes qui cherchent à répondre plus explicitement aux questions posées par les organisateurs de ce débat transatlantique. Comme tout essai voulant en quelques pages offrir un tableau d'ensemble des caractéristiques de la géographie actuelle, celui d'Orlando Peña suscitera sans doute les critiques de ceux qui ne pourront y situer leur propre expérience de notre commune histoire et de notre mémoire collective. II reste que cette représentation très personnelle des «sept piliers de la géographie" méritait d'être versée dans le tronc commun du débat. Adrien Bérubé propose un examen de conscience aux géographes après avoir bien établi, qu'en effet, la géographie a changé. A chacun de répondre. L'essai de L. Deshaies a la valeur d'un témoignage qui garde, volontairement sans aucun doute, le ton que pourrait prendre un étudiant particulièrement doué, soumis au feu roulant d'un enseignement et d'expériences de recherche dont les tenants et les aboutissants restent extrêmement variés et qui sent le besoin de faire le point pour trouver à son tour sa propre voie, et définir son propre projet scientifique, si possible dans une continuité. C'est en regard de ce type de besoin que les géographes auraient intérêt à regarder de plus près, de manière évidemment aussi critique que possible, le travail d'un Lakatos ou d'un Giddens par exemple.

La continuité correspondrait-elle à la redécouverte de l'objet régional comme une pierre angulaire de l'interprétation géographique? Anne Gilbert a raison de poser la question, tout particulièrement dans le contexte du débat qui nous était proposé. Même si son titre dépasse le contenu de son article. Anne Gilbert a su, en tout cas, éviter les pièges des vaines redites en greffant heureusement son plaidoyer sur l'acquis, tout de même considérable, de plus de vingt ans de pratique disciplinaire sur les différents fronts théoriques possibles de la spatialité produite, vécue et reproduite dans le rapport général du social à la matérialité. Elle illustre bien la possibilité de 
réunir en un tout cohérent, "metathéorique ", l'apport des différentes problématiques, et l'occasion qu'en donnent les préoccupations régionales et régionalisantes. Je ne peux alors que partager son point de vue quant aux difficultés pratiques de l'exercice et me féliciter de la finesse des distinctions qu'elle propose dans l'évaluation des difficultés. Le texte reste cependant optimiste et plus que tout autre dans le lot, il peut être considéré comme un possible - parmi d'autres -, "Agenda géographique " au sens où l'entendait Allen Scott à propos de l'étude du rapport «industrialisationurbanisation ».

Autre forme de recherche de la continuité que celle que nous offre $O$. Soubeyran, plus ambitieuse sans doute au plan épistémologique, mais plus dérangeante aussi. Un texte qui veut faire raisonner et dans lequel il faut entrer. Faut-il le considérer à son tour comme cette auberge espagnole qu'il évoque à propos des textes fondateurs? Comme dans tous ses autres travaux, $O$. Soubeyran sollicite du lecteur une attention totale. II a le sens de la circularité. Mais le suivre dans les représentations qu'il nous donne de ce que peut être aujourd'hui la quadrature du cercle ("Brother, can you paradigm?») géographique est gratifiant. D'autant plus qu'en chemin les remarques pertinentes foisonnent et sont parfois frappées comme des médailles. Il a bien raison par ailleurs de relier les conditions de la connaissance géographique à celles de la planification et c'est peut-être par ce biais que ses conclusions, finalement, ne sont pas très éloignées de celles d'Anne Gilbert, encore qu'elle s'inscrive dans une interprétation plus large de l'histoire de la pensée scientifique et philosophique. A ce propos, s'il faut vraiment se prononcer pour savoir si, oui ou non, il y a eu « rupture épistémologique », la définition qu'il en donne me paraît pertinente : nous sommes bien passés du « fait donné » au « fait construit", et c'est bien là une conquête essentielle, à la base de notre entrée en épistémologie et de notre parcours aux configurations progressivement plus endogènes, à la recherche d'un nécessaire dépassement dans un modèle d'autonomie relative. Quant à sa notion de "communauté normale ", comment ne pas l'accueillir avec intérêt, d'autant plus qu'elle renvoie presque directement à l'étude de cette "épistémologie populaire » chère à Vincent Berdoulay et dont les stratégies planificatrices ne sont pas exemptes, tant s'en faut, et qui renvoient, de gré ou de force, à une interrogation sur les lieux du discours.

Finalement, l'approche contextuelle de l'histoire de la pensée géographique dont Vincent Berdouley nous rappelle, si besoin était, les mérites, remet, comme on dit en Suisse, les pendules à l'heure et l'Église au centre du village. Mais l'illustration de la diversité des modalités discursives et de son importance pour mieux comprendre à la fois l'insertion sociale des géographes et le cours complexe de leur pensée est ici heureusement dépassée dans un sens extrêmement positif : la diversité comme atout plutôt que comme problème. Dès le départ, l'hypothèse est forte qui veut que les genres du discours, ces «modèles d'écriture», permettent de rendre compte du pluralisme au sein de notre discipline. Mais évoquer un lien entre particularités discursives et société, langage et demande "sociétale" (on retrouve peut-être ici une des préoccupations d'Adrien Bérubé) est plus ambitieux encore. II était temps de poser, finalement de manière fort simple, les problèmes en ces termes. Qu'on est loin ici des préoccupations qui ont au départ accroché notre attention. II reste que toute "pratique de la géographie humaine", quel que soit son référentiel théorique global, réclamera "les mots pour la dire" et qu'il n'est pas de metalangage, ni «meta-meta langage" sans langage tout court et que l'être de la géographie en dépend d'abord. Pourquoi ne pas reprendre ici, comme mot de la fin, celui de Claude Pouliot à propos de la conception scientifique du paysage terrestre: "seule une opération de langage permet...". J'ajouterai : d'être capable de relier les grandes mutations actuelles à une 
lecture géographique du monde susceptible de montrer que les choix spatiaux qui se présentent à la planète sont aussi des enjeux pour la société et pour la personne et, dans le langage de Jean Remy, que trois niveaux doivent être mis en perspective réciproque, le niveau morphologique, le niveau structuro-fonctionnel, le niveau socioaffectif.

Puis-je répéter ici ce que j'ai voulu montrer dans ma propre contribution à ce débat, en accord finalement avec la majorité des contributions "américaines " ? Dans l'étude des enjeux spatiaux et de l'ordre, de la complexité et des significations, toujours plurielles, du monde, il y a bien des entrées possibles. Les contributions «américaines" en administrent encore une fois la preuve, "post-moderne» peut-être, si ce concept renvoie bien à la mobilité de l'interprétation, corrélative de «l'éclatement des référents dans la pensée d'aujourd'hui ».

\section{Claude RAFFESTIN (Université de Genève)}

Tous les textes ont, bien évidemment, retenu mon attention mais puisque les commentaires doivent être brefs, je me concentrerai plus spécialement sur deux d'entre eux: celui de W. Donald McTaggart (la géographie moderne et la pensée écologique) et celui de Claude Pouliot (Géomorphologie et rupture épistémologique).

Avant de dire à McTaggart tout le bien que je pense de son texte, je voudrais d'abord le «taquiner " un peu sur des raccourcis auxquels je n'adhère pas parce qu'ils caricaturent à l'excès une époque. Je veux parler du deuxième paragraphe de la première page. Si Kant, Hegel et Herder eurent une influence philosophique sur la géographie dont on pourrait abondamment discuter pour en extraire le "bon grain et l'ivraie", je ne crois pas du tout qu'il faille leur assimiler Humboldt, Ritter et Ratzel et surtout qu'il faille imputer à ces derniers une pure tendance «descriptive ». Si Humboldt, Ritter et Ratzel avaient davantage influencé la géographie elle aurait acquis beaucoup plus tôt un statut scientifique dans l'exacte mesure où ils furent les porteurs de modèles, disons de crypto-modèles. D'ailleurs, il est dangereux de faire ce type d'opposition entre $X \mid X^{e}$ et $X X^{e}$ siècles car la plupart des modèles originels de la géographie sont issue de l'effort du XIX siècle.

Cela dit, je crois que l'apport de McTaggart est d'une grande importance lorsqu'il pose le problème des rapports de la géographie avec la pensée écologique. II aurait pu d'ailleurs, à cette occasion rappeler l'œuvre d'un américain du XIXe , G.P. Marsh, qui dans un texte fameux, Man and Nature, a dénoncé d'un point de vue écologique soit deux ans avant l'invention du terme écologie par Haeckel (1866), les dégradations subies par la planète sous l'effet de l'action anthropique. Faut-il dire que je partage tout à fait les remarques de McTaggart sur la nécesstié de la pensée écologique et que je me félicite de le voir mettre en évidence des auteurs comme Georgescu-Roegen et Bateson? Cependant je m'étonne de le voir postuler une géographie écologique qui, naturellement, serait différente de la géographie positiviste, de la géographie humaniste et de la géographie marxiste. Je m'étonne car je crois qu'une des faiblesses de la géographie est justement de s'ajouter des adjectifs. II n'y a toujours pas de géographie mais un nombre croissant de "géographies" suivies d'un adjectif épithète. C'est davantage qu'un sujet d'étonnement pour moi c'est un sujet de presque désespoir car cela veut dire que nous n'avons pas réussi à constituer une discipline mais que nous 
sommes face à un "archipel » disciplinaire. Comment et quand parviendrons-nous à élaborer un système qui assure la continuité entre toutes ces "îles " sans doute belles et utiles en elles-mêmes mais incapables de mettre en commun leur potentiel pour réaliser une synergie? II ne suffit pas, en effet, de créer des champs disciplinaires soustendus par des philosophies spécifiques ni non plus de poser des problématiques capables de susciter de nouvelles méthodes, faut-il encore être capable de se poser la question des relations à établir avec les autres lieux de la géographie. Pour prendre une métaphore politique on a l'impression que la géographie est dans la situation du Saint Empire romain germanique avant l'unité allemande c'est-à-dire divisée en " principautés ", "villes-États " et "petits royaumes ". Nous avons insuffisamment songé à l'intégration. Je crois à l'intégration de la pensée écologique dans la géographie pas à la création d'une géographie écologique.

Le second texte, celui de Pouliot, est particulièrement stimulant et je me réjouis qu'un jeune géographe fasse preuve d'autant d'indépendance d'esprit que de perspicacité. Pour montrer la nécessité d'une " rupture épistémologique " Pouliot illustre son propos par l'exemple du tableau périodique de Mendeleïev « qui a su organiser la chimie en un tout cohérent " en créant un " langage " générateur d'une théorie. Pouliot est préoccupé par le même problème que celui que j'ai évoqué dans le commentaire du texte de McTaggart. Pouliot est un internaliste au sens d'Hilary Putnam qui considère que les relations que l'on établit, en science, doivent l'être à l'intérieur d'un système conceptuel. II postule un système conceptuel tant pour la géomorphologie que la géographie. C'est ce qu'il dit implicitement quand il écrit : « le temps serait venu pour la géomorphologie, et ce à l'exemple de la chimie, de rompre avec la nature". II ne s'agit pas d'établir une correspondance entre des signes et des objets pris dans la nature mais entre des signes et des objets construits. Le rôle qu'il accorde aux formes et aux structures lui fait souhaiter un détour par la "géométrie".

La géographie humaine est dans une position symétrique et on peut se demander si la géographie dans son ensemble n'est pas sous-tendue par une discipline qui n'existe pas, celles des arrangements, des dispositions qu'on peut qualifier de diathétique. Les choses étant, en somme, analogues à celles que Saussure postulait pour la linguistique : la sémiologie, qui était encore à faire, sous-tendait la linguistique et bien d'autres choses encore.

Pouliot illustre, une fois de plus et après beaucoup d'autres, le «principe du détour ": pour comprendre ce qu'on a sous les yeux il faut savoir regarder ailleurs. Les premiers principes de la géographie ont été découverts en regardant les étoiles... Pour comprendre la géomorphologie Pouliot s'intéresse à l'univers...

Je crois qu'il faut remercier McTaggart et Pouliot qui, chacun à leur manière, nous ont montré des "absences" plus ou moins douloureuses dans la géographie contemporaine. Des absences qui nous empêchent de dire qu'il y a une géographie mais qui incitent, indiscutablement, à travailler pour «inventer» un principe de continuité, une sorte d'aimant autour duquel toute la limaille des faits accumulés pourrait s'organiser. Nous savons tous que ce principe de continuité n'est pas cette pseudo-synthèse concomitante au discours, il est dans ce "quelque chose" à construire qui n'est pas encore construit. Ce « quelque chose » qui n'opposera pas les différentes problématiques positiviste, marxiste, humaniste et écologique mais qui les rassemblera non pas dans un tout éclectique mais dans un tout organique. 


\section{Marie-Claire ROBIC (Université de Paris I)}

S'il y a quelque arbitraire à discuter avec seulement une moitié des participants du colloque transatlantique, du moins son éloignement permet-il de mieux réfléchir à quelques écueils de l'exercice. Par exemple, pour revenir à la possibilité d'une " maîtrise de l'histoire" (celle de la géographie) sur laquelle on nous a interrogés, ces textes donnent à lire une considérable hétérogénéité des expériences de géographes et du même coup des "histoires" de la géographie récente. Alors, la fécondité du bilan réflexif, du témoignage, de l'anamnèse ? Faisons son sort à l'idée que l'on reconstruit l'histoire d'une science grâce à la résurrection d'un "oublié ", grand ou petit : le filon a été suffisamment exploité au cours des vingt dernières années pour que l'on rappelle et la fécondité et les limites de cette démarche historiographique militante... Éliminons aussi la croyance en la véracité du témoignage personnel, de l'histoire de vie: la géographie n'est pas affaire de collection de biographies, mais effet de composition, rencontre de champs - et ceci est encore à construire.

Pourtant, pour comprendre où l'on en est, cela aide de retrouver les traces de ceux que la mémoire collective a enfouis, des réflexions et des pratiques perdues, dont l'oubli apparaît comme autant de lapsus collectifs : à ce titre, les propos théoriques et les analyses d'O. Soubeyran surl' " imaginaire disciplinaire » sont extrêmement précieux. De l'histoire récente de la géographie, j'observerais d'abord que l'échantillon " américain " en parle peu, comme avec distance: les "vingt ans de turbulence " seraient-ils une mauvaise mesure ici? C'est ce que suggère par exemple $O$. Peña, qui renvoie au célèbre texte de Schaefer, ainsi que W.D. McTaggart, L. Deshaies et je partage leur attention aux années 1950. Par contre d'autres textes soulignant des scansions plus récentes révèlent la variété des intervenants, tant par leur âge que par la nature du cursus qui les rassemble sur la terre "américaine».

A. Bérubé propose une appréciation d'ensemble du changement, dans la «vie " et dans la géographie. Mais son analyse historique, stimulante, permet-elle de conclure? II relève des mutations socio-politiques entre 1977 et 1987 d'une part, compare par ailleurs deux "lustres» des Annales géographiques (France et USA, 1961-65 et 1981-85) : avec les années soixante et le milieu des années soixante-dix ne confronte$t$-il pas des périodes dissemblables; et les deux revues ont-elles assumé les changements dans la discipline? Quant à l'usage de la notion de paradigme en géographie, elle mériterait discussion. $O$. Soubeyran la mène avec bien des nuances; la position de V. Berdoulay me paraît trop expéditive. Par ailleurs les géographes français ne semblent pas avoir usé et abusé de la notion comme l'auraient fait les géographes "américains " en mal de changement ou de légitimation. Ils ne se sont guère référés à Kuhn pour représenter leurs " combats" au cours des années 1970 contre la géographie classique française: les nouvelles géographies sont défendues au nom des épistémologies de Bachelard, d'Althusser, de Popper, de Foucault, ou d'Habermas..., généralement sous le signe de la rupture épistémologique, du passage de l'idéologie, ou du mythe, ou de l'âge préscientifique, à la science - ce qui n'a guère à voir avec la substitution d'un paradigme à un autre au cours d'une transition entre deux phases de "science normale ": ni les références invoquées ni l'analyse des enjeux n'étaient du même ordre.

Ces remarques n'auraient aucun intérêt si elles ne permettaient de restituer la singularité des processus par lesquels la géographie change ici et là, et de se demander dans le même mouvement, si elle change, pourquoi ici (et pas ailleurs) : de s'interroger sur les configurations qui produisent l'inégale précocité, l'inégale diffusion, et le contenu des changements dans la discipline. Or ce que suggèrent ces textes, dans ce qu'ils disent et dans ce qu'ils rappellent, c'est que pour ce côté de l'Atlantique, 20 ans de 
changement n'est pas une bonne mesure. Pour la France, plus significatif me semble le tournant par lequel l'École géographique française, qui existait il y a encore 20 ou 30 ans, s'est scindée en des courants où beaucoup se reconnaissent plus d'atomes crochus avec tels géographes d'outre-atlantique et d'ailleurs qu'avec leur collègue du bureau voisin. Par contre aussi, si l'on peut penser que la géographie n'a pas eu la même histoire en France et de ce côté de l'Atlantique depuis l'après-guerre (mais je pense, comme je le rappelle dans mon texte et m'en suis expliquée auparavant, qu'une même configuration apparue dans les années 1940-1950 a ébranlé l'épistémologie de la géographie dans toutes les écoles existantes), il semble qu'une convergence plus grande apparaisse aujourd'hui, à travers les défis dont font état les textes de M. Santos, J.L. Klein, D. Harvey et A. Scott.

Les considérations sur la "régularité » ou sur la "récurrence» de l'introspection disciplinaire en géographie ne me paraissent pas très pertinentes; de même ne suis-je pas convaincu de l'existence d'un couplage direct entre activité théorique et efficacité pratique (O. Peña). En clair, cette entrée par l'histoire veut souligner la complexité des systèmes de relations qui concourent à la dynamique de la géographie, et que ces contributions ont tendance à réduire : outillage intellectuel, pratiques sociales, nature de l'objet. L'analyse des textes liant la crise des modèles holistiques (ou le retour au local) aux restructurations du système mondial contemporain suggère que les enjeux actuels sont devenus plus similaires d'un côté et de l'autre de l'Atlantique. L'une des questions ne serait-elle pas de savoir construire des modèles complexes qui rendent compte d'une situation spatiale nouvelle : la "mondialisation ", production de différences entre les lieux dans leur relation quasi simultanée? J'en regrette d'autant que l'article de Harvey et Scott ne souffle guère mot d'une recherche critique sur des instruments heuristiques propres, comme sur ces «espaces relatifs» que des pratiques nouvelles, planificatrices, avaient en leur temps suscités. 